\title{
Patients with chronic periodontitis are more likely to develop upper urinary tract stone: a nation-wide population-based eight-year follow up study
}

\author{
I-Shen Huang ${ }^{1,2,3,4}$ ， Sung-En Huang ${ }^{5}$, Wei-Tang Kao ${ }^{6,7}$ ， Cheng-Yen Chiang ${ }^{8}$, To Chang ${ }^{9}$, Cheng-I Lin ${ }^{10}$,

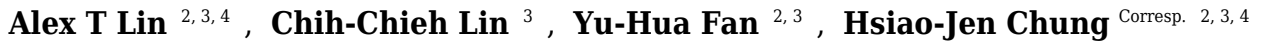 \\ 1 Department of Physiology, School of Medicine, National Yang-Ming University, Taipei, Taiwan \\ 2

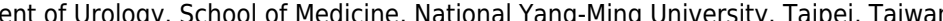 \\ 3 Department of Urology, Taipei veterans general hospital, Taipei, Taiwan \\ 4 Department of Urology, School of Medicine, Shu-Tien Urological Research Center, National Yang-Ming University, Taipei, Taiwan \\ 5 Private practice, Hsinchu city, Taiwan \\ 6 Department of Urology, Shuang Ho Hospital, Taipei Medical University, New Taipei city, Taiwan \\ 7 Graduate Institute of Clinical Medicine, Taipei Medical University, New Taipei city, Taiwan \\ 8 Division of urology, surgical department, Taoyuan General hospital, Ministry of health and welfare, Taoyuan, Taiwan \\ 9 Division of urology, surgical department, National Yang Ming University hospital, Yilan, Taiwan \\ 10 Private practice, Houston, Texas, United states of America \\ Corresponding Author: Hsiao-Jen Chung \\ Email address: hjchung@vghtpe.gov.tw
}

Background The purpose of this study was to investigate the relationship between chronic periodontitis (CP) and upper urinary tract stone (UUTS) in Taiwan by using a population-based data set.

Methods A total of $16,292 \mathrm{CP}$ patients and randomly selected 48,876 controls without chronic periodontitis were selected from the National research database and studied retrospectively. Subjects selected have not been diagnosed with UUTS previously. These subjects were prospectively followed for at least eight years. Cox regression models were used to explore the connection between risk factors and the development of UUTS.

Results The CP patients have a greater chance of developing UUTS compared to controls (1761/16292, $10.8 \%$ vs $4775 / 48876,9.8 \%$, p-values < 0.001$)$. Conditioned logistic regression suggested $\mathrm{CP}$ increases the risk of UUTS development (HR 1.14, 95\% Cl, 1.08-1.20, $\mathrm{p}<0.001$ ). After respective adjustment for age, gender, hypertension and diabetes, results showed that $\mathrm{CP}$ still increases the risk of developing UUTS (HR 1.14, $95 \% \mathrm{Cl}=1.08-1.20, \mathrm{p}<0.001$ ).

Conclusion By using population based database with a minimum eight year follow-up of CP in Taiwan, we discovered patients with CP are more likely to develop UUTS. 
1 Original article

2 Patients with chronic periodontitis are more likely to develop upper

3 urinary tract stone: a nation-wide population-based eight-year follow up

4 study

5 I-Shen Huang ${ }^{1,2,3,4}$, Sung-En Huang 5 , Wei-Tang Kao ${ }^{6,7}$, Cheng-Yen Chiang ${ }^{8}$, To Chang ${ }^{9}$, Cheng-

6 I Lin ${ }^{10}$, Alex T. Lin ${ }^{2,3,4}$, Chih-Chieh Lin ${ }^{3}$, Yu-Hua Fan ${ }^{2,3}$, Hsiao-Jen Chung ${ }^{2,3,4}$

7

$8{ }^{1}$ Department of Physiology, School of Medicine, National Yang-Ming University, Taipei,

9 Taiwan.

$10 \quad{ }^{2}$ Department of Urology, School of Medicine, National Yang-Ming University, Taipei, Taiwan

$11{ }^{3}$ Department of Urology, Taipei Veterans General Hospital, Taipei, Taiwan.

$12{ }^{4}$ Department of Urology, School of Medicine, Shu-Tien Urological Research Center, National

13 Yang-Ming University, Taipei, Taiwan

145 Private practice, Hsinchu city, Taiwan

$15{ }^{6}$ Department of Medical Research, Shuang Ho Hospital, Taipei Medical University,

16 New Taipei City, Taiwan

$17{ }^{7}$ Graduate Institute of Clinical Medicine, College of Medicine, Taipei Medical University, New

18 Taipei City, Taiwan

$19{ }^{8}$ Division of urology, surgical department, Taoyuang General hospital, Ministry of health and

20 welfare, Taoyuang, Taiwan

$21{ }^{9}$ Division of urology, surgical department, National Yang Ming University Hospital, Yilan,

22 Taiwan

$23{ }^{10}$ Private practice, Houston, Texas, USA

*Correspondence author. Dr. Hsiao-Jen Chung, Department of Urology, Taipei Veterans General

27 Hospital, No 201, Section 2, Shipai Rd, Taipei 112, Taiwan, ROC.

Tel: $+886-2-2875-7519 \quad$ Fax: $+886-2-2875-7540$

29 E-mail: hjchung@vghtpe.gov.tw 


\section{Abstract}

32 Background

33 The purpose of this study was to investigate the relationship between chronic periodontitis (CP)

34 and upper urinary tract stone (UUTS) in Taiwan by using a population-based dataset.

35 Methods

36 A total of 16,292 CP patients and randomly selected 48,876 controls without chronic

37 periodontitis were selected from the National research database and studied retrospectively.

38 Subjects selected have not been diagnosed with UUTS previously. These subjects were

39 prospectively followed for at least eight years. Cox regression models were used to explore the

40 connection between risk factors and the development of UUTS.

41 Results

42 The CP patients have a greater chance of developing UUTS compared to controls (1761/16292,

$4310.8 \%$ vs 4775/48876, 9.8\%, p-values < 0.001 ). Conditioned logistic regression suggested CP

44 increases the risk of UUTS development (HR 1.14, 95\% Cl, 1.08-1.20, p < 0.001). After

45 respective adjustment for age, gender, hypertension and diabetes, results showed that $\mathrm{CP}$ still

46 increases the risk of developing UUTS (HR 1.14, 95\% Cl $=1.08-1.20, \mathrm{p}<0.001$ ).

47 Conclusion

48 By using population based database with a minimum eight year follow-up of CP in Taiwan, we

49 discovered patients with CP are more likely to develop UUTS. 
56

57

58

59

60

61

62

63

64

65

66

67

68

69

70

71

72

73

74

75

76

77

78

79

80

81

82

83

84

85

\section{Introduction}

Urolithiasis is common urological disease with prevalence ranging from $5 \%$ to $20 \%$, and varies with gender, age, race and geographical region (Scales et al., 2012; Hall et al., 2009; Stamatelou et al., 2003). The incidence has increased in the US in the last three decades and for the Taiwanese population, an increase of 8.9\% in men between 1998 to 2010 (Huang et al., 2013). Recurrence rate was $6-10 \%$ within one year and up to $35-50 \%$ during a five year follow up (Huang et al., 2013; Pearle et al., 2005; Penniston et al., 2011). The high prevalence and recurrence rate represent a significant cost burden to the healthcare system, especially for the population in their 40 s to 50 s, where the annual expenditure is estimated to exceed $\$ 5$ billion, including direct (treatment) and indirect (work productivity) cost (Penniston et al., 2011; Saigal et al., 2005).

Chronic periodontitis (CP) is another prevalent inflammatory disease of tooth-supporting tissue, caused by oral bacterial infection and resulting in dysregulation of host immunoinflammatory response. In the United States, adults aged 30 years or older have an estimated prevalence of $45 \%$ and CP is positively correlated with increased age and male gender (Eke et al., 2012). In CP, action of matrix degrading enzymes and lipopolysaccharide from oral bacteria trigger the aggregation of an interleukin and tumor necrosis factor, which amplifies the inflammatory response and reduces the damage repair ability of fibroblast, leading to osteoclastogenesis activation and subsequent alveolar bone damage (Armitage et al., 2003; Schenkein et al., 2006). In addition, during the progression and development of CP, bone metabolism and bone remodeling are altered and the marker of bone metabolism could be used as reflection of its progression (Yoshihara et al., 2009). In patients with severe periodontitis, the levels of parathyroid hormones (PTH) were higher and levels of 25(OH)D were lower, indicating that compared to normal subjects, CP patients may have more serious bone resorption and demineralization (Schulze-Späte et al., 2015).

Due to elevated PTH in severe CP patients, which possibly brings hypercalcemia and clinical symptoms such as urolithiasis, we hypothesize that CP can potentially induce urolithiasis. This is this first reported study exploring the relationship between CP and urolithiasis. We analyzed the connection between initial chronic periodontitis and subsequent risk of urolithiasis during the eight-year follow up period, with a nationwide population database. 


\section{Materials and Methods}

88 The study was performed according to the Taipei Veterans General Hospital approved

89 Institutional Review Board protocol (TPEVGH IRB number: 2018-03-003CC).

90 Data Source

91 The National Health Insurance (NHI), launched by the department of Health in Taiwan, has 92 prospectively collected original data for reimbursement, and, since the end of 1996, over $95 \%$ 93 of the Taiwanese population are receivers of health care services. The NHI performs validations 94 of medical charts to ensure the accuracy of documented diagnoses. The official longitudinal 95 dataset derived from the Taiwan NIH, Longitudinal Health Insurance Database 2000 (LHID 2000), 96 contains all the original claim data of 1,000,000 beneficiaries enrolled in year 2000, randomly 97 sampled from the year 2000 Registry for Beneficiaries of the NHI research database. The LHID 2000 has no record of statistically significant difference in gender distribution, age nor healthcare cost between the enrollees from the LHID 2000 and the original NHI research database.

101

\section{Selected cases and controls}

103 From 1997 to 2001, a total of 371,185 subjects without previous diagnosis of urinary stones 104 (ICD-9-CM codes 592 "calculus of the kidney and ureter", 592.0 "calculus of the kidney", 592.1 "calculus of the ureter" or 592.9 "urinary calculus, unspecified" and chronic periodontitis (ICD9-CM codes 523.4 "chronic periodontitis", 523.5 "periodontitis", 523.8 "other specified periodontal diseases" or 523.9 "unspecified gingival and periodontal disease") were enrolled for a minimal follow-up of eight years. Of the study population, 16,292 subjects aged 30-70 years (8,237 males and 8,055 females) developed chronic periodontitis during the follow-up period and served as the study group. The definition of upper urinary tract stones (UUTS) was

111 present in the ICD-9-CM codes: $592.0,592.1$ or 592.9 at least twice, whereas the definition of

112 chronic periodontitis was identified as ICD-9-CM code 523.4 with the additional procedure 113 codes 91006C, 91007C, 91009B or 91010B.

114 Proven risk factors of urinary stones, such as hypertension (ICD-9 codes 401-405) and 115 diabetes mellitus (ICD-9 codes 250), were included into the multi-variable analysis. To increase 116 the validity of our study, subjects with a diagnosis of hypertension and/or diabetes mellitus for 117 at least 3 times within one year were enrolled. 
118 A comparison cohort without chronic periodontitis during the same period was randomly 119 selected, frequency matched 1:3 ( $n=48876)$ for gender, age and comorbidities (diabetes,

120 hypertension, hyperlipidemia). By the end of 2009 , all subjects were followed up for a minimum 121 of eight years.

\section{Statistical Analyses}

123 The distribution of demographic status and comorbidities, including age, sex, diabetes and

124 hypertension, were compared between the CP and non-CP controls. Continuous variables were 125 evaluated by student's $t$ test, and categorical variables were analyzed by the Pearson chi-square 126 test. We compared the study population's characteristics by a crude model to match sex and 127 age, and the crude model was, then, further used again in the adjusted model to match the 128 study population with hypertension and diabetes.

129 Results are represented as hazard ratio (HR) with a corresponding 95\% confidence interval (95\% $130 \mathrm{Cl}$ ). Cox frailty proportional hazard regression models were used to determine the connection 131 between risk factors and the development of upper urinary tract stones. The UUTS-free survival 132 probability in patients regardless of CP matching or hypertension and diabetes during the 133 follow up period was estimated using the Kaplan-Meier method. A log-rank test was applied to 134 generate figure data. We used SAS 9.2 statistics software (SAS Institute Inc., Cary, NC, USA) to 135 analyze data and statistical significance was considered by a conventional $p$ value $<0.05$. 


\section{Results}

140 The demographic and clinical characteristics of the 65,168 subjects and controls, including

14116,292 patients with CP and 48,876 control subjects, are shown in table 1 . Both groups had a 142 similar distribution of sex and age, and were predominantly male (50.6\%), 31-40 years (40.5 143 years) of age.. Diabetes was more prevalent in the CP group at baseline (17.6\% versus $15.9 \%$, $144 \mathrm{p}<0.001)$. Overall, after a minimal follow-up of at least eight years, analysis demonstrates CP 145 subjects have a greater probability percentage for further development of UUTS than the 146 controls (Hazard ratio 1.14, 95\% confidence interval 1.08-1.20, p-values < 0.001), specifically, $1471,761(10.8 \%)$ of the study subjects, and 4,775 (9.8\%) of the controls developed UUTS (Table 2). 148 Table 3 represents the association between UUTS and the subjects' characteristics. Age, sex, 149 diabetes and hypertension were both associated with developing UUTS. (all $p$-values $<0.001$ ). 150 After adjusting for age, gender, hypertension and diabetes, the Cox regression model fitted,

151 based on the study subjects and controls, showed that patients with CP still possess a 152 significantly increased risk of developing UUTS. ( $\mathrm{HR}=1.14,95 \% \mathrm{Cl}=1.08-1.20, \mathrm{p}<0.001)($ Table 153 4). A Kalplan-Meier analysis showed that, during the 13-year study period, the overall 154 cumulative incidence of UUTS was 19.2\% higher for patients with CP than for those who did not 155 have CP $(\mathrm{p}<0.001$, Figure 1$)$.

156 


\section{Discussions}

159 In this nationwide population-based retrospective cohort study, a greater percentage of 160 subjects with CP developed UUTS than that without chronic periodontitis. After adjusting for 161 hypertension and diabetes, regression analysis still pointed to CP as an independent factor for 162 UUTS.

163

164 To date there was no study ever to explore the association between CP and UUTS. It appears 165 that these two diseases share metabolic syndrome (MetS) as a common risk factor, which 166 includes a spectrum of the following medical conditions: dysglycemia, dyslipidemia, visceral 167 obsesity, and hypertension. In longitudinal and cross-sectional studies, there is a positive 168 correlation between MetS and CP --as the number of MetS components in an individual 169 increases, so does the risk of periodontitis. (Kaye et al., 2016; Lee et al., 2015). The postulated 170 mechanism for this association is well studied with dysglycemia, a key feature of MetS. 171 Enhanced production of irreversible advanced glycation end product (AGE) in a hyperglycemia 172 state may result in a defective constitution of extracellular matrix components, resulting in 173 compromised tooth supporting tissue, devastated apparatus and eventual teeth exfoliation 174 (Gurav et al., 2013). In addition, through the induction of IL-1b, TNF-a and PGE2 in dysglycemic 175 state, increased oxidative stress may be associated with an increased expression of the 176 receptor activator of nuclear factor kappaB ligand (RANKL), which plays a critical role in 177 periodontal bone resorption (Wu et al., 2015). In a meta-analysis study studying MetS and 178 urolithiasis, results showed that a higher prevalence of urolithiasis tended to exist among 179 patients with three or more MetS traits(Wong et al., 2016), which is similar to the relationship 180 between MetS and periodontitis. Interestingly, a higher prevalence of uric acid stones and a 181 lower prevalence of calcium phosphate stones tended to exist among patients who have two or 182 more MetS factors, but the relative frequency of calcium oxalate stones remained regardless of 183 the number of MetS factors (Kadlec et al., 2012). A higher frequency of uric acid stones in MetS 184 patients can possibly be explained as consequence of acidic urine, caused by impaired ammonia 185 excretion and increase of endogenous acid production, secondary to insulin resistance 186 (Strohmaier et al., 2012).

188 However, while CP and urolithiasis are both associated with MetS, especially hyperglycemia and 189 hypertension (Wong et al., 2016), after adjusting for diabetes and hypertension, CP remains an 
190 independent risk factor for developing UUTS. Due to the wide spectrum of endocrine and urine 191 abnormalities in urolithiasis patients, it may be more rational to investigate the abnormalities

192 found in chronic periodontitis patients and correlate with pathophysiology of urolithiasis. Given 193 that periodontitis is characterized by tissue and bone destruction, serum calcium level, bone 194 metabolism and, specifically, bone remodeling are altered during the disease progression 195 (Yoshihara et al., 2009; Schulze-Späte et al., 2015). In a recent study, Amarasena et al.,(2008) 196 evaluated 266 Japanese subjects aged 70 years and assessed the relationship of baseline serum 197 calcium and the periodontal disease progression by a follow up of six consecutive years. The 198 finding indicated that subjects with a low serum calcium level have a significantly higher chance 199 of developing periodontal disease. This is corroborated in the Buffalo OsteoPerio study, which 200 followed the periodontal condition in 1,025 postmenopausal women for 5 years, and concluded 201 that women with severe periodontitis and osteoporosis may exhibit accelerated bone loss over 202 time (LaMonte et al., 2013). A higher rate of recurrent periodontitis can also be expected in 203 subjects with osteoporosis after periondontal treatment compared to individuals with normal 204 skeletal bone marrow density (BMD) (Gomes-Filho et al., 2013). Nonetheless, in another 205 observational, prospective study evaluating men 65 years and older, although lower vitamin D and higher level of PTH were observed in severe periodontitis patients, these measures were not associated with progression. Additionally, bone metabolism markers were not associated with periodontis severity, but men with baseline lower levels of bone remodeling markers tended to have a greater improvement in periodontitis (Schulze-Späte et al., 2015). On the other hand, the pathophysiological mechanisms for urolithiasis are complex and diverse,

211 including hypercalciuria, hyperuricosuria, hypocitraturia, hyperoxalateuria and abnormalities in 212 urine $\mathrm{pH}$ (Pak, 1991). The mineral composition of stones is calcium oxalate or calcium 213 phosphate in $80 \%$ of urolithiasis patients, whereas uric acid, struvite and cystine stone 214 represent the rest. Formation of the crystal stone can happen from supersaturation of these 215 crystalline material in urine, which is common when daily urine output is less than 2 liters 216 (Ratkalkar \& Kleinman, 2011). Hypercalciuria is the most common abnormal finding among 217 calcium urolithiasis patients, found in $30-60 \%$ of them, and may result in supersaturation of 218 urinary calcium salts (Pak et al., 1980). In hypercalciuric kidney stone formers, osteoporosis, by 219 means of T-score $<-2.5$, is a prevalent finding. Moreover, hypercalciuria is prevalent in 220 postmenopausal women, and also represents an important predictor for low bone mass 221 (Giannini et al., 2003). Altogether, bone loss or presence of low bone mineral density is a link 
222 both connected to PD and urolithiasis, which could be the explanation found for PD increasing

223 the chance of further UUTS, but this hypothethized biological model needs to be examined by

224 further prospective study evaluating bone mineral density in PD patients with an occurrence of

225 urolithiasis. The mechanism for this finding is, however, beyond the scope of this paper.

227 In our study, we only enrolled patients between $30-70$ years, which is the vast majority of the 228 working population in Taiwan. Therefore, these findings cannot be extrapolated to subjects in 229 different age ranges. Male to female ratio is 1.69. In further UUTS occurrence in our study, this 230 ratio is higher in correspondence with previous Taiwanese population studies, in which the 231 ratio is 1.55 after age adjustment, and also greater than that for the US population reported 232 (Huang et al., 2013; Penniston et al., 2011).

233

234 Although this study detected an association of chronic periodontits with subsequent UUTS 235 development, aided by the use of national database, our results need to be carefully intepreted 236 by knowing their limitations. First, the data were obtained from the NHI database; therefore, 237 certain patient information, including blood pressure and serum glucose, cannot be obtained, 238 which makes the diagnosis less accurate than those made through standardized procedures. 239 Second, other confounding factors such as personal health behavior, diet, lifestyle, height, 240 weight, waistline measurements, drinking and cigarette smoking could not be adjusted in the 241 NHIRD administrative data set. Lastly, we identified patients by diagnostic code (ICD-9), and 242 increased the accuracy of the UUTS diagnosis by the presence of the ICD code twice. The 243 accuracy of chronic periodontitis diagnosis was increased by additional procedure codes. 244 However, we cannot evaluate the severity of disease and the composition of UUTS. Further 245 prospective randomized study may be needed to elucidate the findings of this study and 246 investigate the cause of UUTS in patients with chronic periodontitis.

247 


\section{Conclusion}

249 By using population based database, with a minimal eight year follow-up, of chronic

250 periodontitis in Taiwan, we discovered patients with CP are likely to develop UUTS. CP patients

251 receiving treatment for periodontitis should also be given the body of knowledge about its

252 connection with further development of UUTS.

253 


\section{References}

255 Amarasena N, Yoshihara A, Hirotomi T, Takano N, Miyazaki H. Association 256 between serum calcium and periodontal disease progression in non-

257 institutionalized elderly. Gerodontology. 2008;25(4):245-50. Epub

258 2008/03/31. doi: 10.1111/j.1741-2358.2007.00211.x. PubMed PMID:

25918380783.

260 Armitage GC, Research SiaTCotAAoP. Diagnosis of periodontal diseases. J

261 Periodontol. 2003;74(8):1237-47. doi: 10.1902/jop.2003.74.8.1237.

262 PubMed PMID: 14514240.

263 Eke PI, Dye BA, Wei L, Thornton-Evans GO, Genco RJ, CDC Periodontal 264 Disease Surveillance workgroup: James Beck (University of North Carolina

265 CH, U. S. A.), Gordon Douglass (Past President, American Academy of

266 Periodontology), R.y Page (University of Washin. Prevalence of periodontitis 267 in adults in the United States: 2009 and 2010. J Dent Res.

268 2012;91(10):914-20. Epub 2012/08/30. doi: 10.1177/0022034512457373.

269 PubMed PMID: 22935673.

270 Giannini S, Nobile M, Dalle Carbonare L, Lodetti MG, Sella S, Vittadello G,

271 Minicuci N, Crepaldi G. Hypercalciuria is a common and important finding in 272 postmenopausal women with osteoporosis. Eur J Endocrinol.

273 2003;149(3):209-13. PubMed PMID: 12943523.

274 Gomes-Filho IS, Oliveira TJ, Passos JS, Cerqueira EeM, da Cruz SS, Barreto 275 ML, Coelho JM, Trindade SC, Santos CA, Sarmento VA. Effect of 276 osteoporosis on periodontal therapy among post-menopausal women.

277 Gerodontology. 2013;30(1):40-8. Epub 2012/03/16. doi: 10.1111/j.1741278 2358.2012.00643.x. PubMed PMID: 22420371.

279 Gurav AN. Advanced glycation end products: a link between periodontitis

280 and diabetes mellitus? Curr Diabetes Rev. 2013;9(5):355-61. PubMed PMID: 28123845077. 
282 Hall PM. Nephrolithiasis: treatment, causes, and prevention. Cleve Clin J 283 Med. 2009;76(10):583-91. doi: 10.3949/ccjm.76a.09043. PubMed PMID: $284 \quad 19797458$.

285 Huang WY, Chen YF, Carter S, Chang HC, Lan CF, Huang KH. Epidemiology 286 of upper urinary tract stone disease in a Taiwanese population: a 287 nationwide, population based study. J Urol. 2013;189(6):2158-63. Epub 288 2013/01/09. doi: 10.1016/j.juro.2012.12.105. PubMed PMID: 23313204.

289 Jounai N, Kobiyama K, Takeshita F, Ishii KJ. Recognition of damage290 associated molecular patterns related to nucleic acids during inflammation 291 and vaccination. Front Cell Infect Microbiol. 2012;2:168. Epub 2013/01/08. 292 doi: 10.3389/fcimb.2012.00168. PubMed PMID: 23316484; PubMed Central 293 PMCID: PMCPMC3539075.

294 Kadlec AO, Greco K, Fridirici ZC, Hart ST, Vellos T, Turk TM. Metabolic 295 syndrome and urinary stone composition: what factors matter most? 296 Urology. 2012;80(4):805-10. Epub 2012/07/13. doi:

297 10.1016/j.urology.2012.05.011. PubMed PMID: 22795374.

298 Kaye EK, Chen N, Cabral HJ, Vokonas P, Garcia RI. Metabolic Syndrome 299 and Periodontal Disease Progression in Men. J Dent Res. 2016;95(7):822-8.

300 Epub 2016/03/29. doi: 10.1177/0022034516641053. PubMed PMID:

30127025874 ; PubMed Central PMCID: PMCPMC4914866.

302 LaMonte MJ, Hovey KM, Genco RJ, Millen AE, Trevisan M, Wactawski-Wende 303 J. Five-year changes in periodontal disease measures among 304 postmenopausal females: the Buffalo OsteoPerio study. J Periodontol. 305 2013;84(5):572-84. Epub 2012/07/19. doi: 10.1902/jop.2012.120137. 306 PubMed PMID: 22813344.

307 Lee KS, Lee SG, Kim EK, Jin HJ, Im SU, Lee HK, Merchant AT, Song KB, 308 Choi $\mathrm{YH}$. Metabolic syndrome parameters in adolescents may be 309 determinants for the future periodontal diseases. J Clin Periodontol. 
310 2015;42(2):105-12. Epub 2015/01/20. doi: 10.1111/jcpe.12338. PubMed

311 PMID: 25469423.

312 Pak CY. Etiology and treatment of urolithiasis. Am J Kidney Dis.

313 1991;18(6):624-37. PubMed PMID: 1962646.

314 Pak CY, Britton F, Peterson R, Ward D, Northcutt C, Breslau NA, McGuire J,

315 Sakhaee K, Bush S, Nicar M, Norman DA, Peters P. Ambulatory evaluation

316 of nephrolithiasis. Classification, clinical presentation and diagnostic criteria.

317 Am J Med. 1980;69(1):19-30. PubMed PMID: 6247914.

318 Pearle MS, Calhoun EA, Curhan GC, Project UDoA. Urologic diseases in

319 America project: urolithiasis. J Urol. 2005;173(3):848-57. doi:

320 10.1097/01.ju.0000152082.14384.d7. PubMed PMID: 15711292.

321 Penniston KL, McLaren ID, Greenlee RT, Nakada SY. Urolithiasis in a rural

322 Wisconsin population from 1992 to 2008: narrowing of the male-to-female

323 ratio. J Urol. 2011;185(5):1731-6. Epub 2011/03/21. doi:

324 10.1016/j.juro.2010.12.034. PubMed PMID: 21420112; PubMed Central

325 PMCID: PMCPMC3280819.

326 Ratkalkar VN, Kleinman JG. Mechanisms of Stone Formation. Clin Rev Bone 327 Miner Metab. 2011;9(3-4):187-97. doi: 10.1007/s12018-011-9104-8.

328 PubMed PMID: 22229020; PubMed Central PMCID: PMCPMC3252394.

329 Saigal CS, Joyce G, Timilsina AR, Project UDiA. Direct and indirect costs of

330 nephrolithiasis in an employed population: opportunity for disease

331 management? Kidney Int. 2005;68(4):1808-14. doi: 10.1111/j.1523-

332 1755.2005.00599.x. PubMed PMID: 16164658.

333 Scales CD, Smith AC, Hanley JM, Saigal CS, Project UDiA. Prevalence of

334 kidney stones in the United States. Eur Urol. 2012;62(1):160-5. Epub

335 2012/03/31. doi: 10.1016/j.eururo.2012.03.052. PubMed PMID: 22498635;

336 PubMed Central PMCID: PMCPMC3362665. 
337 Schulze-Späte U, Turner R, Wang Y, Chao R, Schulze PC, Phipps K, Orwoll

338 E, Dam TT. Relationship of Bone Metabolism Biomarkers and Periodontal

339 Disease: The Osteoporotic Fractures in Men (MrOS) Study. J Clin Endocrinol

340 Metab. 2015;100(6):2425-33. Epub 2015/04/09. doi: 10.1210/jc.2014-

341 4180. PubMed PMID: 25856210; PubMed Central PMCID: PMCPMC4454801.

342 Stamatelou KK, Francis ME, Jones CA, Nyberg LM, Curhan GC. Time trends

343 in reported prevalence of kidney stones in the United States: 1976-1994.

344 Kidney Int. 2003;63(5):1817-23. doi: 10.1046/j.1523-1755.2003.00917.x.

345 PubMed PMID: 12675858.

346 Strohmaier WL, Wrobel BM, Schubert G. Overweight, insulin resistance and

347 blood pressure (parameters of the metabolic syndrome) in uric acid

348 urolithiasis. Urol Res. 2012;40(2):171-5. Epub 2011/08/25. doi:

349 10.1007/s00240-011-0403-9. PubMed PMID: 21866382.

350 Wong Y, Cook P, Roderick P, Somani BK. Metabolic Syndrome and Kidney

351 Stone Disease: A Systematic Review of Literature. J Endourol.

352 2016;30(3):246-53. Epub 2016/01/22. doi: 10.1089/end.2015.0567.

353 PubMed PMID: 26576717.

354 Wu YY, Xiao E, Graves DT. Diabetes mellitus related bone metabolism and 355 periodontal disease. Int J Oral Sci. 2015;7(2):63-72. Epub 2015/06/26. doi:

356 10.1038/ijos.2015.2. PubMed PMID: 25857702; PubMed Central PMCID:

357 PMCPMC4817554.

358 Yoshihara A, Deguchi T, Hanada N, Miyazaki H. Relation of bone turnover

359 markers to periodontal disease and jaw bone morphology in elderly

360 Japanese subjects. Oral Dis. 2009;15(2):176-81. doi: 10.1111/j.1601-

361 0825.2008.01511.x. PubMed PMID: 19207487.

362 


\section{Table $\mathbf{1}$ (on next page)}

Demographic characteristics of patients with chronic periodontitis and controls 
1 Table 1. Demographic characteristics of patients with chronic periodontitis and controls

\begin{tabular}{|c|c|c|c|c|c|}
\hline & \multicolumn{2}{|c|}{$\begin{array}{l}\text { Patients with CP } \\
\qquad(n=16292)-\end{array}$} & \multicolumn{2}{|c|}{$\begin{array}{l}\text { Controls } \\
(n=48876)\end{array}$} & \\
\hline Variable & Total No. & $\%$ & Total No. & $\%$ & $P$ value \\
\hline Sex & & & & & 1.00 \\
\hline Male & 8237 & $50.6 \%$ & 24711 & $50.6 \%$ & \\
\hline Female & 8055 & $49.4 \%$ & 24165 & $49.4 \%$ & \\
\hline Age & & & & & 0.634 \\
\hline$<31$ & 526 & $3.2 \%$ & 1840 & $3.8 \%$ & \\
\hline $31-40$ & 6592 & $40.5 \%$ & 19654 & $40.2 \%$ & \\
\hline $41-50$ & 5641 & $34.6 \%$ & 16451 & $33.7 \%$ & \\
\hline $51-60$ & 2360 & $14.5 \%$ & 7239 & $14.8 \%$ & \\
\hline $61-70$ & 1173 & $7.2 \%$ & 3692 & $7.6 \%$ & \\
\hline Personal history & & & & & \\
\hline Diabetes & 2870 & $17.6 \%$ & 7782 & $15.9 \%$ & $<0.001$ \\
\hline Hypertension & 4870 & $29.9 \%$ & 14983 & $30.7 \%$ & 0.067 \\
\hline
\end{tabular}




\section{Table 2 (on next page)}

Crude HR for new-onset upper urinary tract stone (UUTS) among patients with chronic periodontitis and comparison controls 
1 Table 2. Crude HR for new-onset upper urinary tract stone (UUTS) among patients with chronic 2 periodontitis and comparison controls

\begin{tabular}{|c|c|c|c|c|c|c|}
\hline New-onset UUTS & \multicolumn{2}{|c|}{$\begin{array}{l}\text { Total Sample } \\
(\mathrm{n}=65168)\end{array}$} & \multicolumn{2}{|c|}{$\begin{array}{l}\text { Patients with CP } \\
(n=16292)\end{array}$} & \multicolumn{2}{|c|}{$\begin{array}{l}\text { Comparison controls } \\
(\mathrm{n}=48876)\end{array}$} \\
\hline 8-year follow up & No. & $\%$ & No. & $\%$ & No. & $\%$ \\
\hline Yes & 6536 & 10.0 & 1761 & 10.8 & 4775 & 9.8 \\
\hline No & 58632 & 90.0 & 14531 & 89.2 & 44101 & 90.2 \\
\hline Crude HR $(95 \%$ CI) & \multicolumn{2}{|c|}{-} & \multicolumn{2}{|c|}{1.14} & \multicolumn{2}{|c|}{1.00} \\
\hline
\end{tabular}

3

4 


\section{Table 3 (on next page)}

Demographic characteristics of subjects with and without new-onset upper urinary tract urolithiasis (UUTU) during follow-up 
1 Table 3. Demographic characteristics of subjects with and without new-onset upper urinary tract 2 stone (UUTS) during follow-up

\begin{tabular}{|c|c|c|c|c|c|}
\hline & \multicolumn{2}{|c|}{$\begin{array}{l}\text { Subjects with } \\
\text { UUTS } \\
(n=6536)\end{array}$} & \multicolumn{2}{|c|}{$\begin{array}{c}\text { Subjects without } \\
\text { UUTS } \\
(n=58632)\end{array}$} & \\
\hline Variable & Total No. & $\%$ & Total No. & $\%$ & $P$ value \\
\hline Age & \multicolumn{2}{|c|}{ Mean $44.8 \pm 9.9$} & \multicolumn{2}{|c|}{ Mean $43.5 \pm 9.7$} & $<0.001$ \\
\hline Sex & & & & & $<0.001$ \\
\hline Male & 4107 & 62.8 & 28841 & 49.2 & \\
\hline Female & 2429 & 37.2 & 29791 & 50.8 & \\
\hline Periodontitis & & & & & $<0.001$ \\
\hline Yes & 1761 & 26.9 & 14531 & 24.8 & \\
\hline No & 4775 & 73.1 & 44101 & 75.2 & \\
\hline Diabetes & & & & & $<0.001$ \\
\hline Yes & 1379 & 21.1 & 9273 & 15.8 & \\
\hline No & 5157 & 78.9 & 49359 & 84.2 & \\
\hline Hypertension & & & & & $<0.001$ \\
\hline Yes & 2591 & 39.6 & 17262 & 29.4 & \\
\hline No & 3945 & 60.4 & 41370 & 70.6 & \\
\hline
\end{tabular}




\section{Table 4 (on next page)}

The risk for upper urinary tract stone among chronic periodontitis, hypertension and diabetes patients in Cox proportional hazard regression 
Table 4. The risk for upper urinary tract stone among chronic periodontitis, hypertension and diabetes patients in Cox proportional hazard regression

\begin{tabular}{|c|c|c|c|c|c|c|}
\hline & Crude HR & $95 \% \mathrm{CI}$ & $P$ value & Adjusted HR & $95 \% \mathrm{CI}$ & $P$ value \\
\hline \multicolumn{7}{|l|}{ Main Effect } \\
\hline \multirow[t]{2}{*}{ Chronic Periodontitis } & 1.14 & $1.08-1.20$ & $<0.001$ & 1.14 & $1.08-1.20$ & $<0.00$ \\
\hline & & & & & & 1 \\
\hline \multicolumn{7}{|l|}{ Comorbidities } \\
\hline \multirow[t]{2}{*}{ Hypertension } & 1.35 & $1.28-1.44$ & $<0.001$ & 1.17 & $1.10-1.25$ & $<0.00$ \\
\hline & & & & & & 1 \\
\hline Diabetes & 1.48 & $1.41-1.55$ & $<0.001$ & 1.42 & $1.34-1.49$ & $<0.00$ \\
\hline
\end{tabular}

Crude HR: relative hazard ratio

Ajusted HR: multivariable analysis including age, sex and cormorbidities 
Figure 1

Probability free of upper urinary tract stone (UUTS) for periodontitis and nonperiodontitis patients 


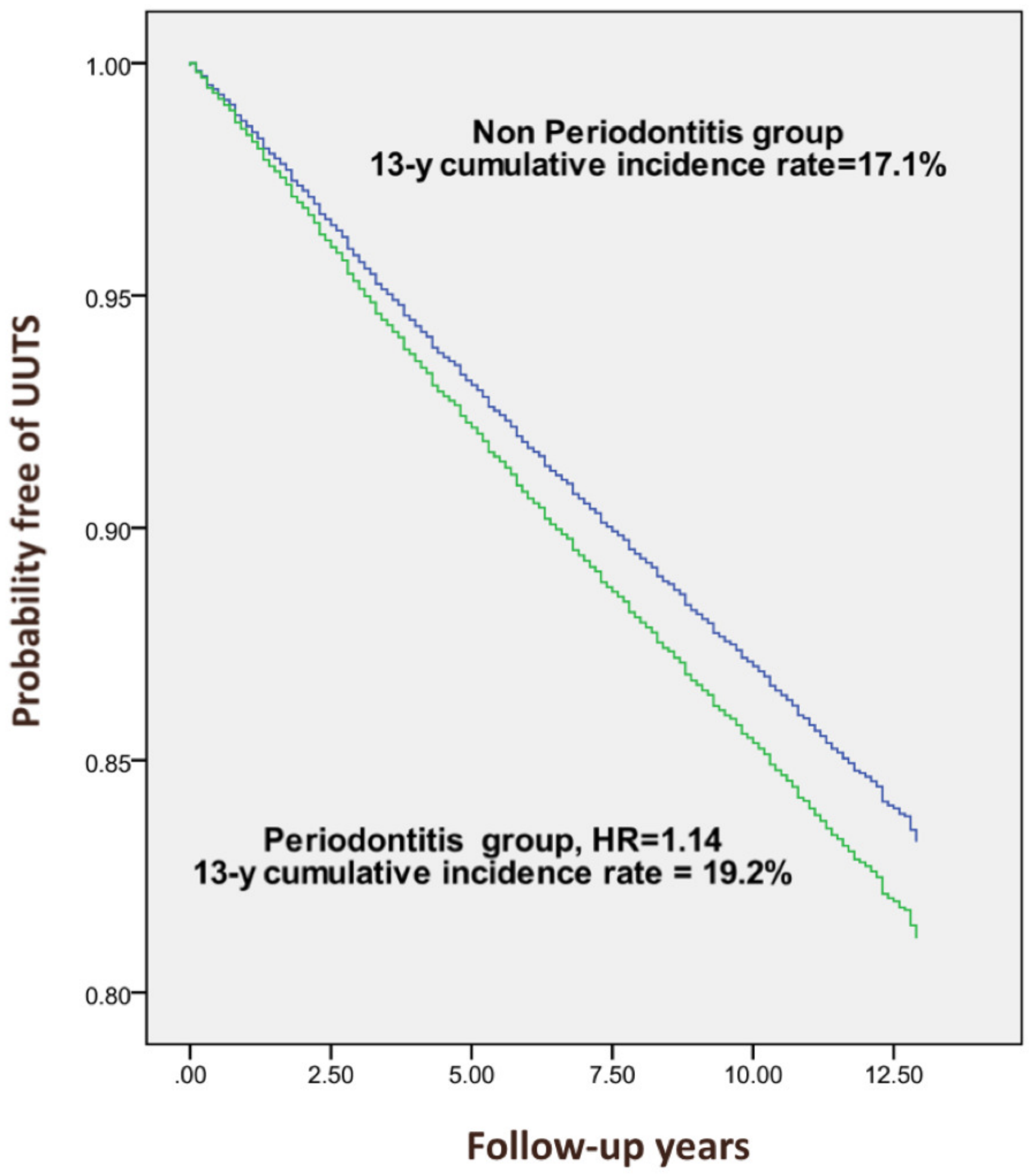

